\title{
Study on the impact of the improvement of traffic accessibility on regional economic disparity-Evidence from the cities along the Beijing-Shanghai HSR
}

Lu Yu ( $\square$ yulusina@126.com)

Shanghai Maritime University https://orcid.org/0000-0003-4644-948X

Yaoyao Wang

Shanghai Maritime University

\section{Research Article}

Keywords: Beijing-Shanghai HSR, traffic accessibility, economic potential, disequilibrium index, economic growth

Posted Date: March 3rd, 2022

DOI: https://doi.org/10.21203/rs.3.rs-1350225/v1

License: (1) This work is licensed under a Creative Commons Attribution 4.0 International License. Read Full License 


\section{Abstract}

Railway transportation, as a major mode of travel in China, has an important impact on traffic conditions and even local economic development. This paper evaluates the changes of traffic accessibility and its impact on regional economic disparity of the cities along the Beijing-Shanghai HSR from 2009 to 2016. On the whole, this paper found that the traffic accessibility of all cities along this line has been greatly improved. Some medium-sized city closer to the core city and located at the middle of the Beijing Shanghai HSR benefit the most, while the small cities farther away from the core cities benefit the least. This line has also brought about a certain improvement on equilibrium level of traffic accessibility, but the increase between the cities is very different. The economic disparity between large and medium-sized cities and small-sized cities along this line has widened.

\subsection{Introduction}

Traffic conditions have been regarded as one of the main impact factors on regional economic development. Railway, as a major mode of transportation, has significant impacts on the traffic conditions of all countries in the world, especially in China. This is because China has a vast territory, a large population and uneven distribution of natural resources. As a fast and economical mode of transportation, railway transportation has a certain advantage in all modes of transportation, and it has become a widely used mode of transportation in China. With the continuous construction of China's railways in recent years, especially the construction of high-speed railway (hereinafter refer as to HSR), the importance and role of railway in China are increasing. For example, during the "12th Five-Year Plan" period in China, the number of people traveling by railway maintained a growth rate of about $10 \%$. In 2016, more than 2.5 billion passengers traveled by rail. By the end of 2016, length of railways in China had reached $124,000 \mathrm{~km}$, of which $17.74 \%$ was HSR. At present, China has the world's second largest railway network after the United States, and the world's largest HSR network and fast rail lines.

In recent years, with the rapid development of China's economy and railway construction, travel time by railway has been greatly reduced between different cities. This large-scale construction of railway will inevitably improve the accessibility level of cities along the line. However, due to many factors such as economic foundation, geographical location, and economic scale and so on, the traffic accessibility for small cities is quite lower than the large and medium-sized cities in China. As the improvement of traffic accessibility will promote the access to economic opportunities, so traffic accessibility is of great significance for regional development. Therefore regional differences in traffic accessibility of railway is regarded as one of the main obstacles to regional coordinated development (one of China's major regional development policy in recent years), and the improvement of transport infrastructure is the main means to reduce this gap. So does development of railway in China really reduce the gap of traffic accessibility of railway between small cities and large and medium-size cities? And does improve of traffic accessibility by railway construction promote the economic growth of cities along the line and reduce regional economic disparities? This paper takes the cities along the Beijing-Shanghai HSR as an example to analyze these issues. This is because the Beijing-Shanghai HSR, known as the "Golden Line" in China's railway network, passes through China's two major economic regions (the Bohai Economic Rim and the Yangtze River Delta). Along this line, there are many important cities in eastern China, not only mega cities such as Shanghai and Beijing, but also some large cities such as Nanjing, Tianjin and Jinan (also provincial capitals), as well as some important medium-sized cities such as Taian, Zaozhuang, etc., and some small cities, such as Suzhou. The Beijing Shanghai HSR is the most representative of all railway lines in China. Therefore, the analysis can partly reflect the impact of China's railway construction on equilibrium degree of traffic accessibility and regional economic growth, which can be seen as an epitome of the overall situation in China.

In recent years, China is committed to achieving the goal of regional coordinated development, so we have to pay more attention to these above impacts. This paper measures the change of traffic accessibility along the Beijing-Shanghai HSR from 2009 to 2016 , and analyses the impact of this change on the economic growth of the cities along the Beijing-Shanghai HSR. These studies will help to understand the characteristics and development of traffic accessibility along the Beijing-Shanghai HSR in recent years, which is of substantial significance for the local government to make policy decisions for the goal of the coordinated development between the regions.

Compared with previous studies (Grant-Muller et al., 2001; Zhang et al., 2016; Chen and Haynes, 2017), this paper contributes to the existing literature from three perspectives: first, previous studies often only studied the impact of HSR on traffic accessibility, but its impact on the equilibrium degree of regional traffic accessibility is ignored. The change of the equilibrium degree of regional traffic accessibility will have a significant impact on future population and economic distribution, so we must pay more attention to it. Second, this paper improves the method of accessibility calculation, which is reflected in the following three aspects: (1) Previous studies often only calculate economic potential and other accessibility indicators based on the minimum travel time, but this is problematic. Therefore the level of accessibility is measured in this paper according to the average travel time. I think this is a more objective and reasonable method to measure accessibility. (2) In the previous calculation methods of economic potential, the potential of the city itself is often ignored, but because the potential of the city itself also plays an important role in the economic growth of the region, it should not be ignored. (3) In previous studies, the impact 
of train frequency between the cities on the economic potential and other accessibility indicators has not been taken into account in the calculation. This paper fully considers this impact and put it into the relevant equations through weighting process. Third, the accessibility calculation in this paper covers a lot of cities, including 212 cities with a resident population of more than 500,000, which basically covers all major cities in China.

The structure of this paper is as follows: the second part is the description of the basic model, the third part is the explanation of data sources and calculation methods, the fourth part is the analysis of result, and the last part is the conclusion.

\subsection{Literature Review And Theoretical Framework}

\subsection{Literature review}

Accessibility analysis is the most common in the investigation of transport infrastructure's impacts on socioeconomic conditions. This is because accessibility is the basic indicator to measure the economic impact of transportation infrastructure (Zhou et al., 2018; Jiao et al., 2020). Morris et al. (1979) defines accessibility as "a condition of spatial segregation of human activities, which represents the degree of difficulty in carrying out economic activities locally and elsewhere". When residents or enterprises can reach their destination to carry out more important activities, the opportunities they get are the concrete embodiment of accessibility. Higher level of traffic accessibility in a region means that local residents will have more opportunities to obtain products from other places or sell local products to other places; it will greatly increase the scale of inter-regional trade and improve the well-being of local residents. Therefore, the change of traffic accessibility has an important impact on the evolution of economical difference between regions and reorganization of regional economy (Vickerman, 2017).

Railway is usually considered to have an important impact on regional economy, because railway lines make cities more closely integrated. Regional economy can benefit from the reduction of transport costs, thus promoting the economic growth of cities along the line, and also reducing the regional economic gap.

Many scholars hold the above viewpoint. As early as the 1920s, Marshall (1920) pointed out that the increase of traffic accessibility in a certain area will improve the ability of the region to provide products and services to other areas, which will lead to the expansion of the market scale in the region, thus improves the degree of regional specialization and production efficiency in the region. Hay (1993) believes that accessibility can be regarded as an indicator of welfare, because the level of individual welfare is related to their convenience in obtaining corresponding services, so the improvement of accessibility enlarges the scope of market choices for individual consumption. Grant-Muller et al (2001), taking the European Union as an example, points out that the European HSR network plays an important role in reducing regional disparities within the EU and strengthening economic and social cohesion.

According to these viewpoints, traffic accessibility is regarded as an important factor affecting the quality of life of local residents (Schurmann et al, 1997). This shows that traffic accessibility is very important to regional economic growth. The poor economic development level in some regions is mainly due to the low level of traffic accessibility. (Lopez et al, 2008).

However, some other scholars hold the opposite point of view. They believe that the improvement of transportation infrastructure will lead to the redistribution of regional economic resources to a large extent, and the regions with larger economies will benefit from it, while those with smaller economies will suffer from it. As early as the 1920s, Hotelling (1929) pointed out that if small cities have to avoid the local market being flooded into the commodities from large cities, there would be a certain barrier between the two. Similarly, Krugman (1991) used the model of New Geographic Economics(NEG) to prove that if there is a moderate reduction in transport costs, economic activities will converge to regions with larger markets under the influence of the Home Market Effect (HME), thus bringing "agglomeration effect" for these regions. For those areas where economic resources are mainly drained, the moderate decline in transport costs has brought significant "siphon effect" for these areas. Under the mechanism of "sharing", "matching" and "learning", this agglomeration effect will further increase the productivity of large cities, thus driving the rapid development of these areas (Graham et al, 2012). This agglomeration effect shows that the intermediate reduction of transport costs leads to the flow of economic resources to the core area, which leads to the deepening of regional economic imbalance.

However, this situation is not unchangeable. The New Geographic Economics also considers that if the transportation cost drops dramatically, the importance of geographical location to enterprises will decrease, which can restore the advantages of peripheral enterprises, while the negative externalities of core areas, such as congestion, pollution and high crime rate, became limitations to further improving economic development of core areas. With the dramatic reduction of transportation costs, for some enterprises, the core region is not suitable to continue to stay. They will move to the peripheral areas, resulting in the "spillover effect" to the peripheral areas. Therefore, the significant reduction of transportation costs will lead to significant changes in regional economic distribution, thus changing the

Page $3 / 16$ 
absolute and relative economic position of peripheral areas, which will lead to the reducing of regional economic disparities. This shows that the relationship between transportation cost and regional economic disparity presents an inverted U-curve, that is, the decrease of transportation cost is often beneficial to the core cities in the regions with larger demand at first, but with the further decline of transportation costs resulting by improvement of traffic accessibility, the economic disparity will gradually narrow, even the small cities farther away from the core cities also can get more rapid development.

In recent years, with the continuous construction of China's railway, the research on China's railway is increasing. Scholars hold similar above viewpoints on the impact of railway on China's regional economy. Meng X et al. (2012) studied the effects of HSR stations on county development in China by applying the PSM-DID method. The study found that HSR construction is conducive to the economic growth of the site area, with a promotion effect of $14 \%$. And the primary source of this promotion effect is a resource redistribution effect in areas located within 30-110 km (26 min) from the station. Liang Yet al.(2020), taking the Guangdong-Guangxi-Guizhou High-speed railway as an representative, uses NPP-VIIRS remote sensing data from the period 2012-2017 and applies the PSM-DID method to analyze the impact of HSR on regional economic growth along the route. The study found that the opening of GGGHSR has a more significant positive impact on less developed areas, counties belong the Guangxi Province and counties outside the one-hour traffic circle. Jia S et al. (2017) used DID and PSM-DID methods to study the economic impact of HSR. The study showed that China's high-speed rail construction has a positive effect on economic growth, but the effect is different for different high-speed rail lines. The role of high-speed rail is mainly depends on whether a location has the necessary conditions to achieve the desired effect. Jin et al. (2020) used a spatial econometric model to analyze the impact of HSRs on economic disparity between 2002 and 2016 in China. The results show that HSRs make a significant positive contribution to economic growth in large-mega cities and developed cities but have insignificant effects in other cities, so HSRs can widen economic disparity in China. Cascetta E et al.(2020) analyzed economic, transport accessibility and social impacts induced by HSR services in Italy after ten years of operation (2008-2018) along the north-south axis Turin-Salerno. The results show that HSR contributed to a significant increase in trainability for the zones along the HSR network, while only marginal for others. And HSR has decrease regional equity in terms of travel time accessibility of $11 \%$, which increase the difference between the zones served by HSR and those not. Luo H. Zhao S. (2021) analyzes the spatial distribution and the spatial inequality of intercity accessibility in the highway network and HSRhighway intermodal network in Liaoning Province, China. The study found that cities in the central areas have better accessibility than peripheral areas, and cities in the peripheral areas and near the HSR lines obtain more advantages from HSR.

\subsection{Model of economic potential and regional economic growth}

There are many methods to measure traffic accessibility. Since the concept of accessibility was put forward in the 1950s, its theoretical basis is developing all the time, resulting in a series of calculation methods. Among them, there are three most common indicators to measure accessibility: weighted average time, economic potential and daily accessibility. However, among the three accessibility indicators, the indicators used to evaluate the impact of traffic accessibility on regional economy are limited to economic potential. This is because economic potential can be used not only as an indicator of traffic accessibility, but also as a measure of the ability of a city's economic activities to provide products and services to the outside world, that is, the potential market capacity of a city or region (Rice et al, 2006), so it can be regarded as a direct factor affecting regional economic growth.

Applying the accessibility index of economic potential, Rice et al (2006) found that the economic potential of a region increases with the improvement of infrastructure, which will greatly promote the improvement of regional labor productivity. The results show that if the economic potential of a region in the UK doubles, the average productivity of workers in that region will increase by $3.5 \%$ under the same conditions. Crucially, even if some businesses and individuals in the region do not travel themselves, they will benefit from this improvement in efficiency. This situation is also suitable for China. Jin et. al. (2013) estimated the average employee productivity in the region by $10 \%$ if the economic potential doubled based on the county/city panel data of Guangdong Province from 1999 to 2009.

For a long time, although there is a strong correlation between economic potential indicators and regional economic growth, its economic theory has been imperfect. Based on the Dixit-Stiglitz model, the New Geographic Economics prove the influence of economic potential on regional labor price (wage). That is, the relationship between regional labor price and regional overall demand (economic potential) can be expressed by wage equation (Crozet and Koenig, 2005; Roberts M et al.,2012; Bai et al., 2012). The equations are as follows:

$$
W_{i}=\frac{\sigma-1}{\sigma}\left(\sum_{j=1}^{n} Y_{j} \tau_{i j}^{-\sigma} P_{j}^{\sigma-1}\right)^{\frac{1}{\sigma}} \beta_{i}^{\frac{1-\sigma}{\sigma}}
$$


In Eq. (1), $w_{i}$ represents the wage income of the region i, $Y_{j}$ represents the total consumption expenditure of the region, $\tau_{i j}$ represents the transportation cost of the iceberg between region $\mathrm{i}$ and $\mathrm{j}: \mathrm{P}$ is the regional comprehensive price index; $\beta_{i}$ is the labor input of the regional marginal products, i.e. the marginal cost; $\sigma$ is the substitution elasticity of the products; and $n$ is the total number of the regions. In this

equation $\sum_{j=1}^{n} Y_{j} \tau_{i j}^{-\sigma} P_{j}^{\sigma-1}$ is defined by Krugman as economic potential (RMP) (Fujita et. al., 199). Harris (1954) economic potential (HMP) (see Eq. 3) can be used to replace RMP into the wage equation, and GDP can be used to replace regional wage income. In addition, because economic growth theory emphasizes the role of capital, labor and government expenditure on economic growth, these variables need to be added to the empirical analysis as control variables. Therefore, the equation becomes:

$$
\begin{gathered}
\operatorname{lnGDP}{ }_{i t}=\beta_{0}+\alpha_{i}+\beta_{1} \ln M P_{i t}+\beta_{2} \ln K_{i t}+ \\
\beta_{3} \ln L_{i t}+\beta_{5} L n G_{i t}+\beta_{6} \ln t_{i t} * \ln M P_{i t}+\varepsilon_{i t}
\end{gathered}
$$

2

In Eq. (2), the subscript i of each variable represents the prefecture-level city, t represents year, GDP is the regional production of a city, $M P$ is the economic potential in Harris form (HMP), $L$ is the total labor force, expressed by the number of social workers in each city, $K$ is the capital input, measured by fixed assets investment, $G$ is the expenditure of local government, represented by local general financial budgetary expenditure. $\beta_{0}$ is expressed as intercept item; $\beta_{1}$ is the regression coefficient of economic potential, while $\beta_{2}$ to $\beta_{6}$ is the regression coefficient of other control variables, $\alpha_{i}$ is the individual fixed effect of the region; $\varepsilon_{i t}$ is the random error item. All variables in Eq. (2) take natural logarithm, and the regression coefficients represent the corresponding elasticity.

In addition, the construction of railways promotes economic and trade exchanges between cities, which help the core cities to more effectively, thus promote economic spillover to the surrounding cities. Therefore, this paper also adds the cross-product term of the travel time from each city to the nearest core city and economic potential in Eq. (2). It is used to measure the spillover effect from the core city. In this paper, the core city refers to the large city, but not only limited to Beijing and Shanghai. This is because that some small and mediumsized cities along the Beijing-Shanghai HSR (such as Zaozhuang, Suzhou, etc.) are too far away from Beijing and Shanghai, and often have more economic ties with the surrounding regional core cities, instead of mega-cities like Beijing or Shanghai. For these cities, the nearest large cities are chosen as the core cities for them. Therefore, this paper chooses Beijing, Shanghai, Tianjin, Jinan and Nanjing as regional core cities, but because these cities are also included in the analysis, the corresponding interactive terms for these cities are cross-product of their internal travel time and economic potential.

(3) Explanation of economic potential

The equations for calculating economic potential are introduced in the form of gravitation in physics, as follow:

$$
P_{i}=\frac{M_{i}}{T_{i}^{\alpha}}+\sum_{j=1}^{n} \frac{M_{j}}{T_{i j}^{\alpha}} * \frac{\mathrm{f}_{\mathrm{ij}}}{\max \left(\mathrm{f}_{\mathrm{ij}}\right)}
$$

3

In Eq. (3), $P_{i}$ denotes the economic potential value of a node, i.e. the economic potential (HMP) defined by Harris (1954), $T_{i j}$ denotes the transportation cost from a node to the destination through a traffic network, and is often expressed by the distance, cost or time between the two places. This paper chooses travel time by railway between the two cities as $T_{i j}$, because the railway distance between the two places remains basically unchanged in a certain period of time, so it cannot reflect the dynamic change of transportation cost; $\mathrm{n}$ is the total number of nodes other than i, $M_{j}$ is the flow of economic factor between a certain economic center and destination, that is to say, its attraction to the surrounding areas, which can be measured by GDP or total population, etc.. In practical application, it is more common to use the total urban population with a threshold as $M_{j}$, because the larger the population size is, the greater the economic demand is, and the more economic opportunities it provides, and the threshold can be set to exclude cities with less impact (Gutierrez, 2001). This paper also uses the total urban population as $M_{j}$, and sets the threshold as 500,000. $\alpha$ is the distance friction coefficient, usually 1 . This is because if $\alpha$ is greater than 1, the closer the city is, the more weight will be given to it. In addition, the equations mentioned above also include the self-potential of the nodes. Although the external economic potential of some cities is small, their own economic potential is considerable, so self-potential should be included in the calculation process, that is $\frac{M_{i}}{T_{i}^{\alpha}}$. Among them, $M_{i}$ is the population size of i city and $T_{i}$ is the transit time within i city. This paper uses the time of congestion within the city to represent it.

Page 5/16 
In addition, in previous studies, only the reciprocal of the travel time is considered as the weight of the traffic flow between the two cities, but this paper argues that the traffic flow between the two cities is affected not only by the travel time, but also by train frequency between the two cities. Even the travel time between different cities is the same, if train frequency is different, the actual convenience is quite different. Therefore, it is necessary to weigh the travel time considering train frequency. In Eq. (3), train frequency between the two cities is $\mathrm{f}_{\mathrm{ij}}$, and $\max \left(f_{i j}\right)$ is the maximum number of trains between the two cities. Thus, the weights of frequencies can be standardized to be between 0 and 1 .

The index above reflects the economic significance of a city, so the calculation of this index can observe the changes of the potential market capacity of each city. Since four hours per journey is the maximum limit for relatively comfortable travel in one day, this paper also constructs and calculates the economic potential of each city setting a time threshold as four hours, For destination cities that arrive in more than four hours, it will be excluded from the Eq. (3). Because most cities mainly trade with the regions within a relatively short time range, so the four-hour economic potential can more accurately reflect the potential market capacity of each city.

\subsection{Research Scope, Data Sources And Calculation Methods 3.1 Time periods and regional scope of the study}

Since the main purpose of this paper is to study change of traffic accessibility and its impact resulting by railway construction. As large scale railway construction in China mainly begin in 2009, especially the construction of HSR. So this paper selected data from 2009 to 2016 for analysis. In the selection of research samples, due to the availability of data, this paper chooses prefectural-level data for analysis. At present, there are 334 prefecture-level administrative units in China in 2016, but in these prefecture-level administrative units, the cities in some areas are relatively small, which has little impact on the traffic accessibility of China. In addition, the operation of HSR requires larger passenger flow than common railway, so it is difficult for small cities to develop HSR. The goal of China Railway Corporation is to gradually cover cities with a population of more than 500,000, so as to provide more powerful supports for economic and social development and mass travel. According to the provisions of the State Council of China in 2014, it was clearly proposed that cities with a permanent population of less than 500,000 in urban areas regard as small cities. Therefore, according to the "China Urban Statistics Yearbook of 2010", this paper excludes the small cities with a population of less than 500,000 in the municipal districts in 2009 , and then excludes the individual prefecture-level cities, island-type cities and some remote cities that have not railways in 2009 . The total number of prefecturelevel cities included in this analysis is 212 , basically including all China's major cities. The urban population data in this paper are also derived from the population of municipal districts in the "China Urban Statistical Yearbook" of the corresponding year..

The 19 cities that the Beijing-Shanghai HSR passes through are Beijing, Langfang, Tianjin, Cangzhou, Dezhou, Jinan, Taian, Jining, Zaozhuang, Xuzhou, Suzhou, Bengbu, Chuzhou, Nanjing, Zhenjiang, Changzhou, Wuxi, Suzhou and Shanghai (see Fig. 1). However, Jining's HSR station is not in the urban area, but in the county-level city under its jurisdiction. Because this paper analyses the impact of traffic accessibility on the economy of the urban districts along the line, the analysis of this paper excludes Jining. Finally, this paper chooses these 18 cities as the research samples to analyze the changes of traffic accessibility between the 18 cities along the Beijing-Shanghai HSR and 212 major cities in China after the opening of the HSR in 2009, so as to explore the impact of HSR construction on the urban traffic accessibility and its differences along the Beijing-Shanghai HSR. 
Table 1

Basic information of HSR stations along Beijing-Shanghai HSR

\begin{tabular}{|c|c|c|c|}
\hline Station name & Mileage(km) & Station type & Distance to $\mathrm{CBD}(\mathrm{km})$ \\
\hline Beijing South & 0 & Provincial Capital level & 7.56 \\
\hline Lang Fang & 59 & Prefecture level & 1.40 \\
\hline Tianjin West & 110 & Provincial Capital level & 5.40 \\
\hline Tianjin South & 131 & Prefecture level & 14.61 \\
\hline Cangzhou West & 219 & Prefecture level & 8.84 \\
\hline Dezhou East & 327 & Prefecture level & 15.71 \\
\hline Jinan West & 419 & Provincial Capital level & 10.54 \\
\hline Taian & 462 & Prefecture level & 9.05 \\
\hline Qufu East & 533 & County level & 46.92 \\
\hline Tengzhou East & 589 & Prefecture level & 37.07 \\
\hline Zaozhuang & 625 & Prefecture level & 25.82 \\
\hline Xuzhou East & 688 & Prefecture level & 10.28 \\
\hline Suzhou East & 767 & Prefecture level & 25.78 \\
\hline Bengbu South & 844 & Prefecture level & 8.07 \\
\hline Dingyuan & 897 & County level & 15.85 \\
\hline Chuzhou South & 959 & Prefecture level & 13.03 \\
\hline Nanjing South & 1018 & Provincial Capital level & 9.35 \\
\hline Zhengjiang South & 1087 & Prefecture level & 6.22 \\
\hline Danyang Noth & 1112 & County level & 9.31 \\
\hline Changzhou North & 1144 & Prefecture level & 8.11 \\
\hline Wuxi East & 1201 & Prefecture level & 15.21 \\
\hline Suzhou North & 1227 & Prefecture level & 12.44 \\
\hline Kunshan South & 1259 & County level & 3.46 \\
\hline Shanghai Hongqiao & 1318 & Provincial Capital level & 13.97 \\
\hline
\end{tabular}

\subsection{Calculation of railway travel time}

To calculate the economic potential index, it is necessary to determine the travel time of inter-city railway. Since the official websites such as the Ministry of Railway do not have historical data on inter-city travel time, China Railway Publishing House publishes the national railway passenger timetable every year; this paper calculates the travel time of inter-city on the basis of these timetables, which includes not only high-speed trains, but also express trains, common speed trains and so on.

This paper considers many factors to calculate the travel time. In many literatures, only the train's running time between the two cities was considered. However, the travel time should include not only the train's running time, but also the congestion time within the city. In addition, for the cities that cannot be directly arrived, the waiting time for the railway transit also should be included. According to Gutierrez et al. (2001), the equation of congestion time in a city is as follows:

$$
T_{i}=15 * \lg (p o p * 10)
$$


In Eq. 4, $T_{i}$ means the internal travel time within a city (i.e. congestion time of a city), and pop is the total population of the city. In addition, if there is no direct train between the two cities, so the transit time also needs to be taken into account. Therefore, the equation of the minimum railway travel time (T1) in this paper is as follows:

$$
T 1_{i j}=T_{i}+T d_{i j}+T r_{i j}+T_{j}
$$

5

In Eq. (5), $T d_{i j}$ means the fastest travel time by railway between $i$ and $j$ cities. If there is a direct train between two cities, running time of the fastest direct train between the two is denotes as $T d_{i j}$. If the travel between two cities by railway requires to transfer, the accumulation of the minimum travel time between different node cities of the route equal to $T d_{i j} . T_{i}$ denotes the internal travel time within city $i$, and $T_{j}$ denotes the internal travel time within city $j$. $T r_{i j}$ denotes the transit waiting time. With the continuous construction of China's railways, the transit waiting time is greatly shortened. Therefore, this paper assumes that waiting time for one transfer is 60 minutes. If it needs to be transferred several times between two cities by railway, the time needs to be accumulated.

In addition, for the calculation of the travel time by railway between two cities, since multiple types of trains actually runs between cities, including both G-prefix, D-prefix, T-prefix, K-prefix express trains and other common speed trains. Therefore, it is not appropriate to measure the travel time between two cities only by the running time of the fastest train, so this paper also calculates average travel time (T2) by railway between two cities. The equations are as follows:

$$
T 2_{i j}=T_{i}+\sum_{f=1}^{n} T d_{i j} \mid n+T r_{i j}+T_{j}
$$

6

In Eq. $6, \sum_{f=1}^{n} T d_{i j}$ is the accumulation of the running time of all trains between $i$ and $j$, $\mathrm{n}$ represents the total number of all trains between the two, and the average running time by railway between two cities is obtained. Then adding it up with the internal commuting time of two cities and transfer time, we can get the average travel time by railway between two cities.

\subsection{Statistical description of relevant data}

The data in this paper are based on the urban panel data according to China Urban Statistical Yearbook from 2009 to 2016 . Considering that China's central and local governments often conduct administrative division adjustment, but geographical scope of administrative jurisdiction for prefecture level is relative stable, so this paper uses the data at prefecture level for analysis. In addition, as regional GDP, fixed assets investment and other variables are calculated at the prices of the respective years, in order to eliminate the impact of inflation, this paper has made the following adjustments: Taking 2009 as the base year, this paper calculates and adjusts the GDP of each city according to its GDP index to get the real GDP. Similarly, based on the fixed-asset investment prices indices of each province in 2009 (China's statistical yearbook does not report data on the price indices of fixed asset investment at the prefecture level), fixed asset investment in each city belong to same province has been deflated based on above price indices. As government expenditure is also calculated at the prices of the respective years, it is deflated based on the CPI price index of each province taking 2009 as the base year. GDP deflator index is calculated according to the regional GDP index of China Statistical Yearbook (at provincial level) and China Regional Economic Statistics Yearbook (at prefecture level). In these yearbooks, the previous year of the respective years is taken as the base year. The data of fixed-asset investment price indices is from China Price Statistics Yearbook, the data of CPI price index is from China Regional Economic Statistics Yearbook.

For the railway travel time from each city to the nearest core city, according to the above, it can be based on the minimum travel time and the average travel time by railway. In addition, in order to further test the role of economic potential in the economy, this paper uses eight forms of economic potential analysis (see Table 1), including the economic potential calculated on the basis of the minimum railway travel time and the four-hour economic potential (which can be divided into two cases, not including self-potential and including self-potential). The economic potential calculated by average railway travel time is treated in the same way. These types of economic potential are represented by $\mathrm{mpl}$, fourmpl, $\mathrm{m} 1$, fourm1, mp2, fourmp2, $\mathrm{m} 2$, foum2 respectively. The following are descriptive statistics of the main variables. 
Table 2 Descriptive statistics of major variables

\begin{tabular}{|c|c|c|c|c|c|}
\hline variables & observations & mean & $\begin{array}{l}\text { standard } \\
\text { deviation }\end{array}$ & minimum & Maximum \\
\hline Gross regional product $(10,000$ yuan $)(Y)$ & 144 & $4.2 \mathrm{E}+07$ & $5.8 \mathrm{E}+07$ & $1.3 \mathrm{E}+06$ & $2.6 \mathrm{E}+08$ \\
\hline Fixed assets investment $(10,000$ yuan $)(\mathrm{K})$ & 144 & $2.0 \mathrm{E}+07$ & $2.2 \mathrm{E}+07$ & $7.3 \mathrm{E}+05$ & $1.2 \mathrm{E}+08$ \\
\hline Employees (10,000 people) (L) & 144 & 123.56 & 196.16 & 3.70 & 791.52 \\
\hline Government expenditure (10,000 yuan) (G) & 144 & $7.3 \mathrm{E}+06$ & $1.2 \mathrm{E}+07$ & $1.6 \mathrm{E}+05$ & $6.3 \mathrm{E}+07$ \\
\hline \multicolumn{6}{|l|}{ Based on Minimum Railway Travel Time } \\
\hline $\begin{array}{l}\text { Economic Potential (Unit: Millions of people /per } \\
\text { hour, The same below) (mpl) }\end{array}$ & 144 & 9.43 & 7.29 & 0.74 & 31.96 \\
\hline Four hours Economic Potential(fourmpl) & 144 & 8.23 & 7.01 & 0.29 & 30.19 \\
\hline Economic Potential(include self-Potential)(ml) & 144 & 19.66 & 13.38 & 4.15 & 57.40 \\
\hline $\begin{array}{l}\text { Four hours Economic Potential (include self - } \\
\text { Potential)(fourml) }\end{array}$ & 144 & 18.46 & 12.93 & 3.88 & 54.75 \\
\hline $\begin{array}{l}\text { Based on Average Railway Travel Time } \\
\text { Economic Potential(mp2) }\end{array}$ & 144 & 6.39 & 5.27 & 0.61 & 23.96 \\
\hline Four hours Economic Potential(fourmp2) & 144 & 4.91 & 5.11 & 0.16 & 22.09 \\
\hline Economic Potential(include self-Potential)(m2) & 144 & 16.62 & 12.32 & 3.80 & 52.00 \\
\hline $\begin{array}{l}\text { Four hours Economic Potential (include self- } \\
\text { Potential) (fourm2) }\end{array}$ & 144 & 15.14 & 12.06 & 3.36 & 49.34 \\
\hline
\end{tabular}

\subsection{Empirical Results And Analysis}

\subsection{Changes of traffic accessibility and equilibrium level along Beijing-Shanghai HSR}

4.11 Changes of traffic accessibility

This paper calculates the economic potential of the cities along the Beijing-Shanghai HSR according to Eq. 3. The results are shown in Table 2. Due to space limitations, this paper only lists the results of 2009 and 2016 for comparison, and only lists the calculation results including self-potential. The first two columns in Table 2 are calculated based on the minimum railway travel time, and the last two columns are calculated based on the average railway travel time. These results all take into account the impact of train frequency.

As can be seen from Table 2, with the continuous construction of HSR, from 2009 to 2016, the economic potential calculated by the minimum travel time increased by $96.4 \%$ on average, and the four-hour economic potential increased by $93.7 \%$ on average. The economic potential calculated by the average travel time increased by $76.5 \%$ and the four-hour economic potential increased by $70.9 \%$ on average. On the whole, the accessibility level of the cities along the Beijing-Shanghai HSR has risen dramatically under the impetus of the national railway construction, regardless of the calculation criteria.

Table 2 calculates the economic potential of cities based on the minimum travel time. According to the calculation results in Table 2, the greatest change in economic potential is not the regional core cities, such as Shanghai, Beijing, Tianjin and other cities. On the contrary, economic potential of some medium-sized cities have changed greatly, which are two situations as follow: one is some cities are close to core cities (usually the provincial capital), these cities will benefit from the externalities of convenient transportation in big cities. Therefore the traffic accessibility of these cities has been improved a lot, such as Cangzhou, Suzhou, Wuxi and so on; the other is some medium-sized city located at the middle of the Beijing Shanghai HSR, railway construction has greatly shortened the travel time of these cities to other core cities, which gradually reflects the location advantages of these cities, such as Xuzhou, Dezhou and so on. However, for some small cities far away from the core city, such as Suzhou, Zaozhuang and so on, the improvement of their accessibility is limited. This situation is more obvious, especially for the calculation results based on the average travel time. For example, the economic potential of Suzhou base on the above results increased by only $35.5 \%$, while the economic potential of four hours increased by only $22.3 \%$. In addition, for the megacities such as Beijing and Shanghai, because their original traffic accessibility level is high, it is very difficult to continue to improve significantly. Furthermore, the population size of megacities was strictly controlled in recent years, so their economic potential has not increased significantly. 
Table 3

Economic potential of the cities along Beijing-Shanghai HSR based on travel time

\begin{tabular}{|c|c|c|c|c|c|c|c|c|c|c|c|c|}
\hline \multirow[b]{3}{*}{ cities } & \multicolumn{6}{|c|}{ Based on the minimum Travel Time by railway } & \multicolumn{6}{|c|}{ Based on the average travel time by railway } \\
\hline & \multicolumn{3}{|c|}{$\begin{array}{l}\text { Economic Potential(m1) } \\
\text { (Unit: Millions of people } \\
\text { /per hour) }\end{array}$} & \multicolumn{3}{|c|}{$\begin{array}{l}\text { Four Hours Economic } \\
\text { Potential(fourm } 1 \text { ) } \\
\text { (Unit: Millions of people } \\
\text { /per hour) }\end{array}$} & \multicolumn{3}{|c|}{$\begin{array}{l}\text { Economic Potential(m2) } \\
\text { (Unit: Millions of people } \\
\text { /per hour) }\end{array}$} & \multicolumn{3}{|c|}{$\begin{array}{l}\text { Four Hours Economic } \\
\text { Potential(fourm2) } \\
\text { (Unit: Millions of people } \\
\text { /per hour) }\end{array}$} \\
\hline & 2009 & 2016 & change(\%) & 2009 & 2016 & change(\%) & 2009 & 2016 & change(\%) & 2009 & 2016 & change(\%) \\
\hline Beijing & 34.7 & 49.5 & 42.5 & 33.8 & 46.5 & 37.5 & 33.9 & 46.4 & 37.0 & 32.8 & 43.3 & 31.8 \\
\hline Langfang & 5.1 & 9.4 & 84.6 & 4.7 & 8.5 & 80.6 & 4.9 & 8.0 & 62.8 & 4.6 & 7.2 & 57.3 \\
\hline Tianjin & 21.9 & 36.3 & 66.2 & 21.1 & 33.5 & 59.0 & 20.9 & 32.6 & 56.3 & 20.1 & 30.3 & 50.5 \\
\hline Cangzhou & 4.9 & 12.2 & 148.7 & 4.4 & 10.9 & 145.0 & 4.3 & 8.7 & 103.6 & 3.9 & 7.4 & 91.1 \\
\hline Dezhou & 5.4 & 14.0 & 157.0 & 4.8 & 12.2 & 154.2 & 4.6 & 10.7 & 132.5 & 3.7 & 9.2 & 145.5 \\
\hline Jinan & 11.5 & 25.7 & 123.7 & 10.7 & 22.7 & 111.4 & 10.8 & 21.7 & 101.2 & 9.7 & 18.4 & 90.4 \\
\hline Tai'an & 7.2 & 12.2 & 69.2 & 6.3 & 10.6 & 68.1 & 6.6 & 9.6 & 44.5 & 5.9 & 7.8 & 30.9 \\
\hline Zaozhuang & 7.3 & 11.6 & 58.9 & 6.9 & 10.8 & 58.0 & 7.2 & 10.0 & 40.0 & 6.7 & 8.4 & 24.8 \\
\hline Xuzhou & 8.9 & 21.7 & 142.9 & 7.4 & 20.6 & 177.3 & 7.9 & 16.7 & 111.4 & 6.4 & 11.8 & 84.8 \\
\hline Suzhou & 7.0 & 10.8 & 54.4 & 6.4 & 9.8 & 53.7 & 6.6 & 9.0 & 35.5 & 6.0 & 7.4 & 22.3 \\
\hline Bengpu & 7.0 & 13.8 & 96.2 & 5.6 & 12.4 & 123.4 & 5.9 & 9.7 & 63.6 & 4.7 & 6.8 & 45.6 \\
\hline Chuzhou & 4.5 & 9.0 & 97.9 & 4.2 & 7.8 & 85.0 & 4.0 & 6.7 & 68.0 & 3.5 & 4.8 & 37.8 \\
\hline Nanjing & 17.5 & 38.6 & 120.1 & 16.8 & 35.9 & 113.5 & 15.8 & 30.5 & 92.8 & 13.7 & 27.7 & 102.9 \\
\hline Zhenjiang & 8.8 & 22.2 & 152.7 & 8.4 & 21.1 & 152.8 & 7.2 & 16.1 & 123.7 & 5.7 & 14.9 & 160.4 \\
\hline Changzhou & 12.2 & 30.7 & 152.3 & 11.6 & 29.5 & 153.3 & 10.4 & 23.9 & 129.3 & 10.0 & 22.4 & 124.2 \\
\hline Wuxi & 13.3 & 34.9 & 161.9 & 12.8 & 33.5 & 161.9 & 11.4 & 27.0 & 136.9 & 10.9 & 25.6 & 134.0 \\
\hline Suzhou & 13.1 & 38.3 & 193.1 & 12.7 & 36.7 & 189.8 & 11.3 & 31.4 & 179.1 & 10.9 & 29.8 & 174.3 \\
\hline Shanghai & 37.0 & 55.8 & 50.8 & 36.3 & 53.2 & 46.4 & 35.9 & 51.2 & 42.5 & 34.9 & 48.7 & 39.5 \\
\hline mean & 12.6 & 24.8 & 96.4 & 11.9 & 23.1 & 93.7 & 11.6 & 20.6 & 76.5 & 10.8 & 18.4 & 70.9 \\
\hline
\end{tabular}

According to the calculation results based on the minimum travel time and the average travel time by railway, the change of the two is similar except the increase of the latter is relatively small. However, for some cities, there is a big difference between the two kinds of calculation results. For example, for the four-hour economic potential based on the minimum travel time by railway, the three cities with the greatest increase are Suzhou, Xuzhou and Wuxi, but in the calculation results based on the average travel time by railway, the three cities with the greatest increase are Suzhou, Zhenjiang and Dezhou. Except for Suzhou, the order of other cities has changed. This result shows that it should not to assess the change of traffic accessibility only based on the result by the minimum travel time. For example, the minimum travel time between some cities has been greatly shortened, but the average travel time has not increased much, so the real situation is not as significant as the calculation results of the former.

4.12 Changes in the equilibrium degree of traffic accessibility

This paper use two commonly methods to measure the change of the equilibrium degree of traffic accessibility along the Beijing-Shanghai HSR, namely the coefficient of variation and the Gini coefficient. If the absolute change of two coefficients is negative, the equilibrium degree will increase, and vice versa. In this paper, the corresponding calculation is shown in Table 3 and in Table 4.

According to Table 4, it can be found that from 2009 to 2016, no matter what method is adopted, and the degree of equilibrium increased. On the whole, as the calculation of economic potential is a combination of population size and transportation cost. Because the population 
size of the core cities is large, so the economic potential of mega-cities is always at the forefront, but the gap of traffic accessibility between large cities and small-medium cities has narrowed.

Table 4

Non-equilibrium index of traffic accessibility along Beijing-Shanghai HSR

\begin{tabular}{|lccccc|}
\hline & \multicolumn{2}{c}{ Based on Minimum Railway Travel Time } & \multicolumn{2}{c|}{ Based on Average Railway Travel Time } \\
\hline & 2009 & 2016 & Change & 2009 & 2016 \\
\hline Coefficient of variation & & & & & \\
\hline Economic Potential & 0.762 & 0.589 & -0.173 & 0.815 & 0.659 \\
\hline Four Hours Economic Potential & 0.803 & 0.609 & -0.194 & -0.156 \\
\hline Gini coefficient & & & & 0.871 & -0.150 \\
\hline Economic Potential & 0.363 & 0.316 & -0.047 & 0.379 & 0.345 \\
\hline Four Hours Economic Potential & 0.382 & 0.326 & -0.056 & -0.034 \\
\hline
\end{tabular}

In addition, according to the calculation results based on average travel time, the equilibrium degree of economic potential is relatively lower, and its decline is limited, which also shows that it is inaccurate to appraise the change of the equilibrium degree of economic potential only by the calculation result based on the minimum travel time. Although the minimum travel time between many cities has been greatly reduced, the decrease of average travel time is relatively small. Meanwhile, there are always great differences in population size between cities. Therefore, the increase of equilibrium degree of economic potential is not as significant as the result calculated by the minimum travel time.

4.2 The impact of the improvement of traffic accessibility on the economic growth of cities along the line

Based on Eq.2, this paper uses panel fixed effect estimation method to analyze the impact of economic potential (representing the level of traffic accessibility) on the economic growth of cities along Beijing-Shanghai HSR. Hsiao C. (2007) pointed out that panel data contains more information, more changes and less collinearity between variables than cross-section data. In addition, the application of panel data leads to greater degrees of freedom, which enhances the efficiency of regression estimation.

According to the common processing of panel data estimation, Hausman test is carried out at first. The results show that the fixed effect should be adopted (see Table 4), which also conforms to the theoretical basis underlying the economic growth model. Second, in order to deal with the common cross-sectional heteroscedasticity problem, robust standard error is used in this paper. Third, as there is certain collinearity between various forms of economic potential, it is substituted into the model one by one for regression (see Table 4).

Meanwhile, The cross-product term of travel time and economic potential is also substituted into the regression model. For example, TMP1 is the cross-product of $T 1_{i k}$ (the minimum travel time from each city to the nearest core city k by railway, taking natural logarithm) and $m p l$, $T M P 2$ is the product of $T 2_{i k}$ (the average travel time from each city to the nearest core city $\mathrm{k}$ by railway, also taking natural logarithm) and $m p 2$, and so on. Furthermore, in order to control for endogenous problem that are common in economic analysis as much as possible, all kinds of economic potentials are regressed using their first order lag form, and all models control the time fixed effect and regional fixed effect. The estimated results are shown in the table 4. 
Table 4

Estimated results of the impact of traffic accessibility on economic growth.

\begin{tabular}{|c|c|c|c|c|c|c|c|c|}
\hline variables & (1) & (2) & (3) & (4) & (5) & (6) & (7) & (8) \\
\hline \multirow[t]{2}{*}{ K } & $0.214^{\star \star \star}$ & $0.213^{\star \star}$ & $0.202^{\star \star \star}$ & $0.199^{\star *}$ & $0.210^{\star \star}$ & $0.209^{\star *}$ & $0.200^{\star \star \star}$ & $0.209^{\text {*x }}$ \\
\hline & $(0.071)$ & $(0.074)$ & $(0.066)$ & $(0.072)$ & $(0.073)$ & $(0.079)$ & $(0.067)$ & $(0.076)$ \\
\hline \multirow[t]{2}{*}{ L } & $0.150^{\star}$ & $0.140^{\star}$ & $0.113^{*}$ & $0.129^{\star}$ & $0.191^{*}$ & $0.182^{\star}$ & $0.116^{*}$ & $0.164^{*}$ \\
\hline & $(0.117)$ & $(0.119)$ & $(0.109)$ & $(0.113)$ & $(0.116)$ & $(0.122)$ & $(0.111)$ & $(0.119)$ \\
\hline \multirow[t]{2}{*}{ G } & $0.064^{\star \star}$ & $0.061^{* *}$ & $0.052^{* *}$ & $0.060^{* *}$ & $0.079^{\star \star}$ & $0.075^{\star *}$ & $0.058^{* *}$ & $0.082^{\star \star}$ \\
\hline & $(0.025)$ & $(0.025)$ & $(0.022)$ & $(0.025)$ & $(0.027)$ & $(0.026)$ & $(0.024)$ & $(0.030)$ \\
\hline \multirow[t]{2}{*}{ MP1 } & 0.175 & & & & & & & \\
\hline & $(0.129)$ & & & & & & & \\
\hline \multirow[t]{2}{*}{ TMP1 } & 0.011 & & & & & & & \\
\hline & $(0.038)$ & & & & & & & \\
\hline \multirow[t]{2}{*}{ MP2 } & & $0.196^{*}$ & & & & & & \\
\hline & & $(0.103)$ & & & & & & \\
\hline \multirow[t]{2}{*}{ TMP2 } & & 0.009 & & & & & & \\
\hline & & $(0.031)$ & & & & & & \\
\hline \multirow[t]{2}{*}{ M1 } & & & $0.525^{\star \star \star}$ & & & & & \\
\hline & & & $(0.156)$ & & & & & \\
\hline \multirow[t]{2}{*}{ TM1 } & & & -0.010 & & & & & \\
\hline & & & $(0.030)$ & & & & & \\
\hline \multirow[t]{2}{*}{ M2 } & & & & $1.009^{\star \star \star}$ & & & & \\
\hline & & & & $(0.341)$ & & & & \\
\hline \multirow[t]{2}{*}{ TM2 } & & & & -0.116 & & & & \\
\hline & & & & $(0.076)$ & & & & \\
\hline \multirow[t]{2}{*}{ fourMP1 } & & & & & 0.202 & & & \\
\hline & & & & & $(0.146)$ & & & \\
\hline \multirow[t]{2}{*}{ TfourMP1 } & & & & & -0.009 & & & \\
\hline & & & & & $(0.040)$ & & & \\
\hline \multirow[t]{2}{*}{ fourMP2 } & & & & & & $0.133^{\star}$ & & \\
\hline & & & & & & $(0.073)$ & & \\
\hline \multirow[t]{2}{*}{ TfourMP2 } & & & & & & 0.009 & & \\
\hline & & & & & & $(0.022)$ & & \\
\hline \multirow[t]{2}{*}{ fourM1 } & & & & & & & $0.484^{\star \star \star}$ & \\
\hline & & & & & & & $(0.144)$ & \\
\hline \multirow[t]{2}{*}{ TfourM1 } & & & & & & & -0.001 & \\
\hline & & & & & & & $(0.029)$ & \\
\hline
\end{tabular}

note: Robust standard errors in parentheses; ${ }^{* \star *} p<0.01$, ** $p<0.05$, * $p<0.1$ 


\begin{tabular}{|c|c|c|c|c|c|c|c|c|}
\hline variables & (1) & $(2)$ & (3) & (4) & $(5)$ & (6) & (7) & (8) \\
\hline \multirow[t]{2}{*}{ fourM2 } & & & & & & & & $1.311^{\star *}$ \\
\hline & & & & & & & & $(0.465)$ \\
\hline \multirow[t]{2}{*}{ TfourM2 } & & & & & & & & $-0.208^{*}$ \\
\hline & & & & & & & & $(0.098)$ \\
\hline \multirow[t]{2}{*}{ constant } & $11.265^{\star \star \star}$ & $11.427^{\star \star \star}$ & $10.847^{\star \star \star}$ & $10.705^{\star \star \star}$ & $11.093^{\star \star \star}$ & $11.291^{\star \star \star}$ & $10.841^{\star \star \star}$ & $10.336^{\star \star \star}$ \\
\hline & $(1.172)$ & $(1.254)$ & $(0.881)$ & $(0.908)$ & $(1.211)$ & $(1.381)$ & $(0.895)$ & $(0.946)$ \\
\hline With-in $\mathrm{R}^{2}$ & 0.871 & 0.870 & 0.879 & 0.875 & 0.859 & 0.855 & 0.876 & 0.863 \\
\hline F value & 148.218 & 158.254 & 164.509 & 152.553 & 87.081 & 145.943 & 151.611 & 141.925 \\
\hline Hausman & 66.10 & 93.85 & 929.80 & 209.87 & 91.62 & 90.74 & 449.62 & 92.47 \\
\hline Observations & 144 & 144 & 144 & 144 & 144 & 144 & 144 & 144 \\
\hline
\end{tabular}

It can be seen that the coefficients of fixed assets investment are positive and significant in table 4. The coefficients of labor force and local government expenditure are the same as above, which are consistent with the expectations of common economic theory.

First, according to the estimated results in Table 4, the coefficients of economic potential in various forms are positive and significant, which is consistent with the previous expectations. That is to say, the construction of railways will bring about an increase in the speed of railways, improving the potential market capacity of cities along the line and promoting the economic growth of these cities. From an economic point of view, on the one hand, the development of railway greatly save travel time and improve economic exchanges between regions, flow of labor force, capital and other factors of production have become more frequent, more product choice for consumers, deepening regional economic integration, lower transaction costs, enhanced technological exchanges and so on. On the other hand, the development of railways has also increased the total volume of traffic flow between cities, thus expanding the market size of various cities. All of these mentioned above accelerate economic growth of the cities along the line.

Second, for the economic potential calculated by the minimum travel time and the average travel time, the coefficient and statistical significance of the latter are higher than those of the former, which shows that the economic growth is more sensitive to the economic potential calculated by the average travel time, that can truly represent the potential market capacity of each city. Only the decrease of the average travel time between cities can truly reflect the reduction of transportation costs, instead of the reduction of the minimum railway time.

Third, for two forms of economic potentials, which includes own potentials and excludes own potentials, both the coefficients and statistical significance of the former are higher than those of the latter. That is to say, for measuring the impact of economic potentials on economic growth, we cannot neglect self-potential of the city itself. Because the market demand of a region includes not only external demand, but also internal demand, which is also very important. Although some cities are far away from other cities, resulting in low external potential, if their own population and economic scale is large, then their internal potential will also promote rapid economic development.

Fourth, all the cross-product terms are not significant or negative, which indicates that the economic spillover of core cities shows a declining trend with distance increase. It also shows that although some relatively remote cities (such as Suzhou, Zaozhuang and so on) have access to HSR, so the travel time to other cities is greatly reduced, the increase of their accessibility is limited compared with other cities closer to core cities. Therefore, it can be concluded that for these small cities far from the core cities, the development of railway only leads to a moderate reduction in transport costs. Due to the small economic scale and poor economic attraction of these cities, their economic resources tend to flow to the surrounding large cities, and the promotion of these cities on economic growth brought by the development of railways is relatively limited.

\subsection{Conclusions}

Taking the cities along the Beijing-Shanghai HSR as an example, this paper investigates the impact of railway construction on urban traffic accessibility and local economic growth. 
First, it is found that, after the large-scale and continuous construction of China's national railways, the traffic accessibility of all cities along the Beijing-Shanghai HSR has been greatly improved in recent years, among which the mid-sized cities closer to the core city benefit the most, while for the small-sized cities farther away from the core city, the improvement of their accessibility is limited. At the same time, this paper uses some indexes to analyze the change of the equilibrium degree of accessibility along the line. According to the calculation results, it is found that the construction of railway also brings about a certain improvement on the equilibrium level of accessibility, but the increase is not very significant.

Second, the improvement of traffic accessibility also promotes the economic growth of the cities along the Beijing-Shanghai HSR, but the effect on the cities along the line is different. The results of this study show that the area along the Beijing-Shanghai HSR is still in the initial stage of inverted U-shaped curve, and the economic spillover of the central cities shows a declining trend with distance. This shows that the development of railways only brings about a moderate reduction of transport cost on some cities along the line, especially for those smallsized cities farther away from the core cities. In these small cities, their economic resources tend to flow into the core cities instead. Railway development plays a relatively limited role in promoting the economic growth of these small cities. Therefore, based on the ubiquitous phenomenon of economic agglomeration, we should focus on some medium-sized cities located in the middle of Beijing-Shanghai HSR (such as Xuzhou, Jinan, etc.) and stimulate them to become new economic growth pole. Therefore, they can produce strong economic spillover effects on the vast inland areas and promote the coordinated economic development along the Beijing-Shanghai HSR. At the same time, the policy of restricting the development of core cities should be relaxed appropriately. After the core cities continue growing bigger and stronger, thus the spillover effect from them to the peripheral areas will inevitably increase. In addition, we should continue to vigorously promote investment in transport infrastructure, increase road network density, strengthen the process of regional economic integration, and substantially reduce the transportation costs between regions, so as to promote the "spillover effect" of core cities and narrow the regional economic development gap.

Finally, this paper suggests that the average travel time by railway should be used to evaluate the impact of traffic accessibility, instead of the minimum travel time by railway. Moreover, the calculation of economic potential should include its own potential, so as to evaluate the impact of traffic accessibility on regional economic growth more reasonably. At the same time, the impact of train frequency on traffic accessibility should be considered as much as possible during the calculation of traffic accessibility.

\section{Declarations}

Competing interests: The authors declare no competing interests.

\section{References}

1. Grant-Muller, S.M., MacKie, P., Nellthorp, J., et al.: Economic appraisal of European transport projects: the state-of-the-art revisited[J]. Transp. Reviews 21(2), 237-261 (2001)

2. Zhang, W., Nian, P., Lyu, G.: A multimodal approach to assessing accessibility of a high-speed railway station[J]. J. Transp. Geogr. 54, 91-101 (2016)

3. Chen, Z., Haynes, K.E.: Impact of high-speed rail on regional economic disparity in China[J]. J. Transp. Geogr. 65, 80-91 (2017)

4. Zhou, J., Yang, L., Li, L.: The implications of high-speed rail for Chinese cities: Connectivity and accessibility[J]. Transp. Res. Part A: Policy Pract. 116, 308-326 (2018)

5. Jiao, J., Wang, J., Zhang, F., et al.: Roles of accessibility, connectivity and spatial interdependence in realizing the economic impact of high-speed rail: Evidence from China[J]. Transp. Policy 91, 1-15 (2020)

6. Morris, J.M., Dumble, P.L., Wigan, M.R.: Accessibility indicators for transport planning[J]. Transp. Res. Part A 13(2), 91-109 (1979)

7. Vickerman, R.: Can high-speed rail have a transformative effect on the economy? [J]. TransportPolicy,2017,(1):1-7

8. Marshall, A.: Principles of Economics: 8th Edition [M]. Macmillan Press, London (1920)

9. Hay, A.: Equity and welfare in the geography of public transport provision [J]. J. Transp. Geogr. 1(2), 95-101 (1993)

10. Schurmann, C.Spiekermann K,Wegener M.Accessibility indicators[J].Berichteausdemlnstitut fur Raumplanung,1997,39

11. Lopez, E., Gutierrez, J., Gomez, G.: Measuring regional cohesion effects of large-scale transport infrastructure investments: an accessibility approach[J]. Eur. Plan. Stud. 16(2), 277-301 (2008)

12. Hotelling, H.: Stability in Competition[J]. Econ. J. 39(153), 41-57 (1929)

13. Krugman, P.: Increasing returns to scale and economic geography[J]. J. Polit. Econ. 99(3), 483-499 (1991) 
14. Graham, D.J., Melo, P.C.: Assessment of wider economic impacts of high-speed rail for Great Britain[J]. Transp. Res. Record J. Transp. Res. Board 2261(1), 15-24 (2012)

15. Meng, X., Lin, S., Zhu, X.: The resource redistribution effect of high-speed rail stations on the economic growth of neighbouring regions: Evidence from China[J]. Transp. Policy 68, 178-191 (2018)

16. Liang, Y., Zhou, K., Li, X., et al.: Effectiveness of high-speed railway on regional economic growth for less developed areas[J]. J. Transp. Geogr. 82, 102621 (2020)

17. Jia, S., Zhou, C., Qin, C.: No difference in effect of high-speed rail on regional economic growth based on match effect perspective? [J]. Transp. Res. Part A: Policy Pract. 106, 144-157 (2017)

18. Jin, M., Lin, K.C., Shi, W., et al.: Impacts of high-speed railways on economic growth and disparity in China[J]. Transp. Res. Part A: Policy Pract. 138, 158-171 (2020)

19. Cascetta, E., Cartenì, A., Henke, I., et al.: Economic growth, transport accessibility and regional equity impacts of high-speed railways in Italy: ten years ex post evaluation and future perspectives[J]. Transp. Res. Part A: Policy Pract. 139, 412-428 (2020)

20. Luo, H., Zhao, S.: Impacts of high-speed rail on the inequality of intercity accessibility: A case study of Liaoning Province, China[J]. J. Transp. Geogr. 90, 102920 (2021)

21. Rice, P., Venables, A.J., Patacchini, E.: Spatial determinants of productivity: Analysis for the regions of Great Britain[J]. Regional Science and Urban Economics, 2006, 36(6): pp. 727-752

22. Jin, Y., Bullock, R., Fang, W.: Regional impacts of high speed rail in China baseline report fora case study of Yunfu in Guangdong province [J]. World Bank. Other Operational Studies 60(3), 528-532 (2013)

23. Crozet, M., Koenig, P.: The cohesion versus growth tradeoff: Evidence from EU regions(1980-2000)[C]. ERSA Conference Paper No. ersa05p716, 2005

24. Roberts, M., Deichmann, U., Fingleton, B., et al.: Evaluating China's road to prosperity: A new economic geography approach[J]. Regional Science and Urban Economics, 2012, 42(4): pp. 580-594

25. Bai, C.E., Ma, H., Pan, W.: Spatial spillover and regional economic growth in China[J]. China Econ. Rev. 23(4), 982-990 (2012)

26. Fujita, M., Krugman, P., Venables, A.J.: The spatial economy: Cities, regions, and international trade[M]. MIT Press, Cambrige (1999)

27. Harris, C.D.: The Market as a factor in the localization of industry in the United States[J]. Ann. Assoc. Am. Geogr. 44(4), 315-348 (1954)

28. Gutierrez, J.: Location, economic potential and daily accessibility: An analysis of the accessibility impact of the highs-peed line MadridBardelona-French border. Journal of Transport Geography,2001,9(4):229-242

29. Hsiao, C.: Panel data analysis-advantages and challenges [J]. Test 16(1), 1-22 (2007)

\section{Figures}




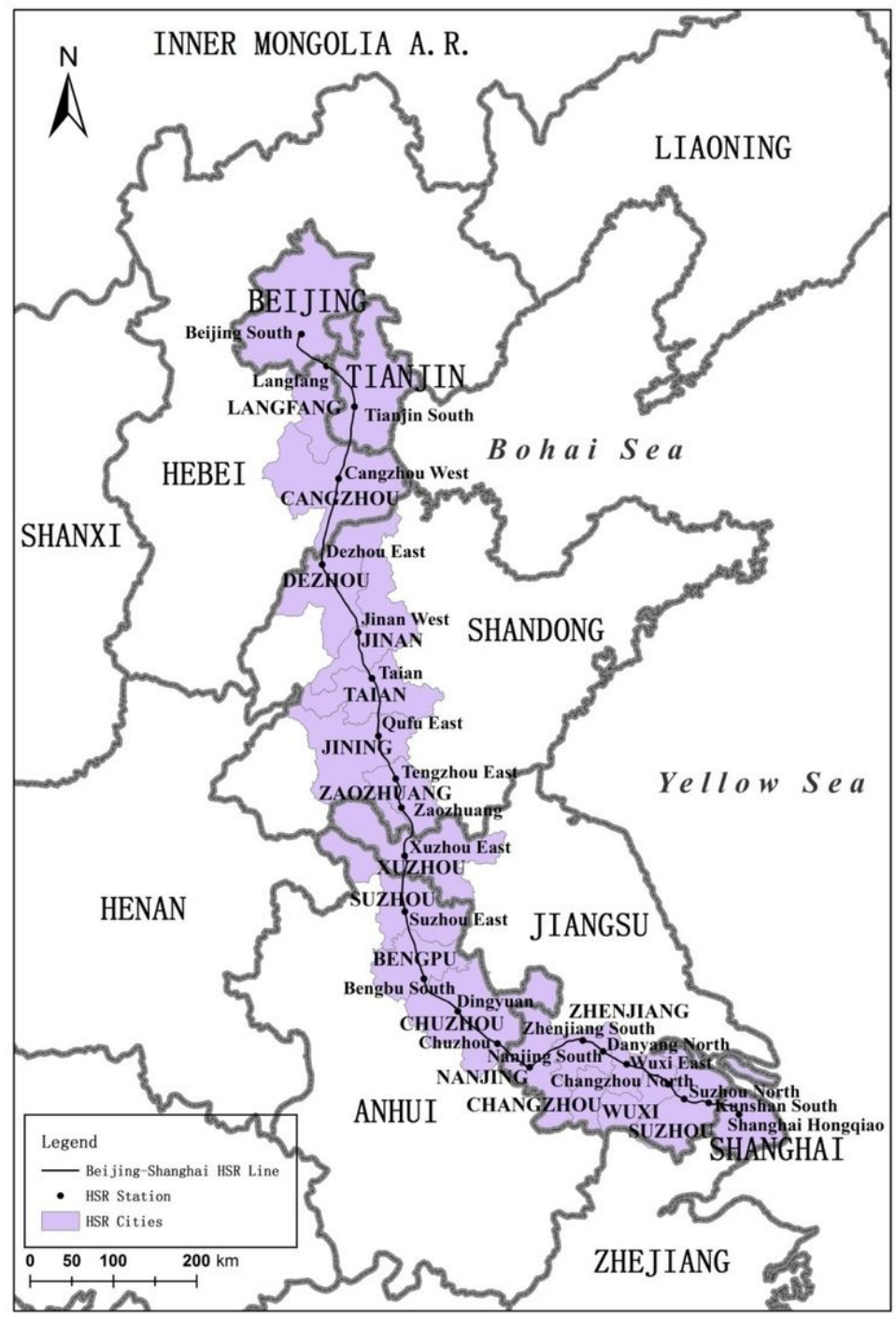

Figure 1

Geographical scope of Beijing-Shanghai HSR

Note: The geographic coordinates of Beijing-Shanghai HSR line and HSR stations are derived from gpsspg website

(http://www.gpsspg.com/). Tianjin West Station is not on the main line of the Beijing-Shanghai HSR, so it is not shown in this figure. 